\title{
Direct and Simple Experimental Crystallographic Method to Calculate Partial Charges with Atomistic Correspondence to ab-initio Methods
}

\author{
P Prakash ${ }^{1}$, A Byrne ${ }^{1}$, T Keller $^{1}$, M Zdilla ${ }^{2}$ \\ ${ }^{1}$ Temple University, Philadelphia, PA, ${ }^{2}$ Chemistry, Temple Univ, Philadelphia, PA \\ prabhat.prakash@students.iiserpune.ac.in
}

Derivation of partial charges in small and large scale molecular systems is important for modeling of various experimental and theoretical properties like dipole moments, auto-correlation functions, charge disparity, understanding of dispersion, benchmark of classical MD simulations and electrostatic potential energy surface mapping. A correspondence between theoretical calculations (based on single/small number of molecules) is usually established with macroscopic IR/Raman spectra or dipole moment measurements. Such comparisons are indirect and lack a fine mapping of electrostatic potential from theory to experiment. In a new approach developed as the experimental part of this work, partial charges are calculated from crystallographic model refinement. The experimental method exhibits a satisfactory correspondence with partial charges obtained using quantum chemistry calculations. Particularly, gas phase partial charges from CHELPG method and condensed phase Lowdin charges correlate well and validate this experimental method.

Acta Cryst. (2020). A76, a194 